\title{
Modelling of the First-Order Time-Varying Filters with Periodically Variable Coefficients
}

\author{
Anna Piwowar and Dariusz Grabowski \\ Faculty of Electrical Engineering, Silesian University of Technology, Ul. Akademicka 10, Gliwice, Poland \\ Correspondence should be addressed to Dariusz Grabowski; dariusz.grabowski@polsl.pl
}

Received 7 March 2017; Revised 26 May 2017; Accepted 31 May 2017; Published 4 July 2017

Academic Editor: Marcello Vasta

Copyright @ 2017 Anna Piwowar and Dariusz Grabowski. This is an open access article distributed under the Creative Commons Attribution License, which permits unrestricted use, distribution, and reproduction in any medium, provided the original work is properly cited.

\begin{abstract}
The article is devoted to modelling and analysis of linear time-varying (LTV) filters with periodically variable coefficients. A transmission model of such filters has been described. Equations expressing the filter response for a given class of periodic parametric functions have been obtained and presented in a closed form. The results have been illustrated by an example.
\end{abstract}

\section{Introduction}

Linear time-varying systems are nonstationary deterministic systems with parameters variable in time. They are also called parametric systems or shortly LTV systems. Those systems are a direct generalization of classical linear and time invariant (LTI) systems. Many theoretical works [1-5] as well as practical applications have been devoted to these systems. In particular, LTV systems can be applied in signal processing [6-11], especially in sampling systems [12], signal filtering and noise reduction $[7,8,13-17]$, and current compensators in power networks $[18,19]$, amplifiers $[5,20]$, chaos generators [21], electromagnetic launchers [22], and medical devices [23]. The main advantages of LTV system application include improvement of the dynamic properties of systems and reduction of the transient state $[16,24]$.

The analysis of LTV systems can be found, among others, in $[5,20,25-30]$. In particular, these works concern parametric systems described by the second- or higher-order differential equations with time-varying parameters. In the literature, there are some methods of LTV system analysis based on transformation of such equations into equations known from the applied mathematics, for example, into Riccati equation $[25,26]$ or Floquet equation [20] as well as Mathieu, Meissner, or Hill equations [5]. These methods require the determination of the state matrix having time-varying elements [20], followed by calculation of the generalized eigenvalues
[27] or Wroński's fundamental solution matrix [27, 28]. This approach is very useful in the case of stability analysis [31, 32] or spectral analysis [27], but it does not usually lead to analytic solutions which are expressed in a closed form.

Analytic solutions to the above-mentioned differential equations exist only in some specific cases which depend strictly on the parametric functions: the waveforms of timevarying parameters. In the theory of differential equations [33], the fundamental solutions of the first-order equation and some second-order equations with time-varying coefficients can be found. On the base of Wroński's fundamental solution matrix [5, 25-28], we can determine solutions to the equations describing LTV systems. The solution of the firstorder equation has a form of an exponential function with an integral of the parametric function in the exponent. On the other hand, the fundamental solutions of the second-order equations are much more complex and can be expressed by special functions of mathematical physics, such as the first- and the second-kind Bessel functions of noninteger orders as well as confluent hypergeometric functions [24, 33]. Determination of a closed form solution for the firstand the second-order systems is possible only if integrals of a combination of fundamental solutions and parametric functions can be calculated.

In the era of applying numerical approach to solving almost everything, it must be reminded that the closed form solutions are important and cannot be ignored or neglected. 
Their importance comes from their role in understanding of qualitative features of phenomena and processes described by differential equations or impulse response functions. Moreover, even if there is no clear physical meaning of a closed form solution, it can still be used to verify the convergence and evaluate the errors of numerical algorithms as well as asymptotic and approximate analytical methods. In the case of the presented model, the numerical solution was also implemented in order to compare the analytical and the numerical results.

The paper shows that, for the first-order LTV system and the assumed class of periodic and exponential parametric functions, it is possible to determine the system impulse response in a closed form for a wide class of excitations, including signals with finite energy or finite average power. The first-order systems can be treated as elementary blocks which allow constructing more complex LTV systems. The determination of the LTV system impulse response is essential as it not only allows calculating the system response to any excitation, but also enables obtaining frequency characteristics of LTV filters. The time waveform of the parametric function, which in our case is interpreted as the time-varying cutoff frequency, has been chosen very carefully. First of all, from the signal theory point of view, exponential changes of the cutoff frequency correspond directly to the dynamic properties of the filter. Moreover, the obtained solution can be extended for a wider class of periodic parametric functions including all the functions which can be expressed by the algebraic sum of exponential functions or can be approximated by such functions with sufficiently small error. Finally, if the parametric function expressing the variability of the coefficient of the first-order differential equation is periodic and exponential, it is possible to find a closed form solution to this equation. According to our knowledge, such closed form solution has not been given in the literature so far.

\section{Transmission Model of LTV Systems}

The transformation rules for systems composed of elementary single input single output LTI blocks are well known in the control theory [34]. In the continuous-time or discrete-time domain, the algebra of these transformations is based on the algebraic sum and the convolution operations $( \pm, *)$ with impulse responses of elementary sections as kernels of these operations. In the domain of $L$ transform or F-transform, algebra is based on the classical definition of the algebraic sum and the multiplication operations $( \pm, \cdot)$ with arguments being the transfer functions or spectral functions of elementary blocks. In the case of LTV systems with coefficients variable in time, the classical definition of operational transfer functions has no sense $[1,35]$.

The transmission model of an LTV system describes an input-output signal relation. The model of a parametric system can be obtained on the basis of the stationary prototype using the algorithm presented in Figure 1.

Based on a known transfer function of the classical filter $H(s)$ (1), by the inverse Laplace transform, one can obtain

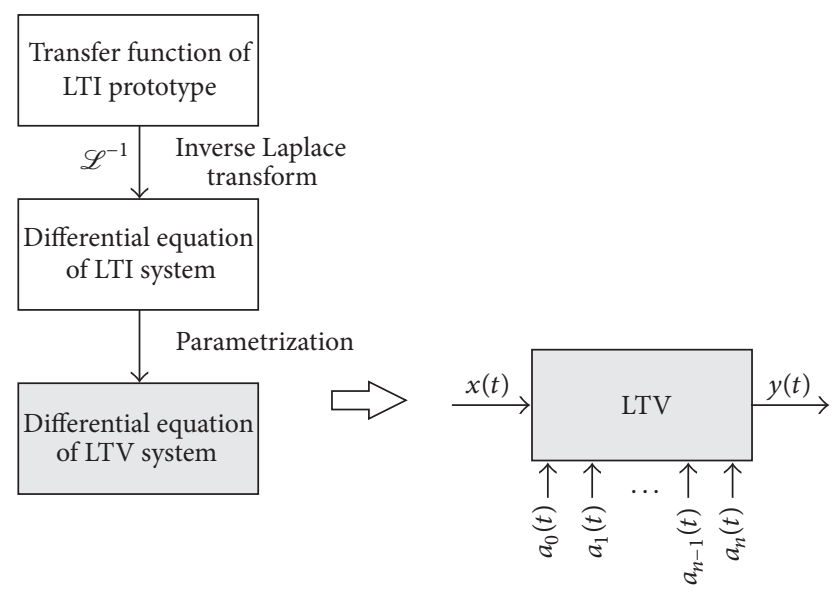

FIgURE 1: Algorithm of LTI to LTV system transformation.

the differential equation of an LTI system (2) with zero initial conditions.

$$
\begin{gathered}
H(s)=\frac{Y(s)}{U(s)}=\frac{b_{n} s^{n}+b_{n-1} s^{n-1}+\cdots+b_{1} s+b_{0}}{a_{n} s^{n}+a_{n-1} s^{n-1}+\cdots+a_{1} s+a_{0}}, \\
a_{k}, b_{k} \in \mathrm{R}, k, n \in \mathrm{N}, \\
a_{n} y^{(n)}(t)+a_{n-1} y^{(n-1)}(t)+\cdots+a_{1} y^{\prime}(t)+a_{0} y(t) \\
=b_{n} u^{(n)}(t)+b_{n-1} u^{(n-1)}(t)+\cdots+b_{1} u^{\prime}(t)+b_{0} u(t),
\end{gathered}
$$

where

$$
\begin{aligned}
& y(t) \text { is the output signal, } \\
& u(t) \text { is the input signal. }
\end{aligned}
$$

Then, after the variation of constant equation coefficients, one can obtain the parametric differential equation:

$$
\begin{aligned}
a_{n}(t) & y^{(n)}(t)+a_{n-1}(t) y^{(n-1)}(t)+\cdots+a_{1}(t) y^{\prime}(t) \\
& +a_{0}(t) y(t) \\
= & b_{n}(t) u^{(n)}(t)+b_{n-1}(t) u^{(n-1)}(t)+\cdots \\
& +b_{1}(t) u^{\prime}(t)+b_{0}(t) u(t) .
\end{aligned}
$$

In the following considerations, it has been assumed that only the parameters on the left side of equation can be varied. Further discussion has been carried out for a section described by (3) with an assumption that the input signal is an equivalent signal $x(t)$. In such a case, the analysis of any parametric section of the $n$th order can be reduced to the analysis of a low-pass LTV section:

$$
\begin{aligned}
a_{n}(t) & y^{(n)}(t)+a_{n-1}(t) y^{(n-1)}(t)+\cdots+a_{1}(t) y^{\prime}(t) \\
& +a_{0}(t) y(t)=x(t) .
\end{aligned}
$$

In the time domain, LTV systems are described in two different ways. The first of them is a linear differential 


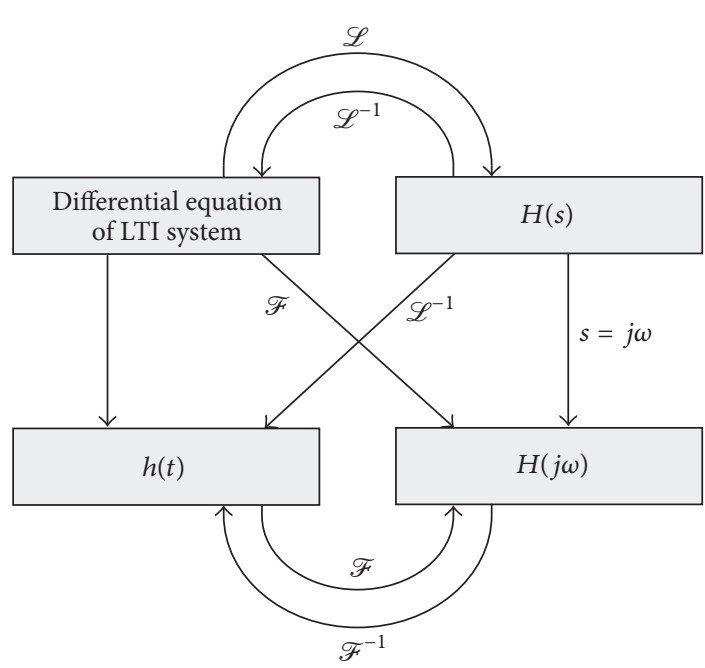

(a)

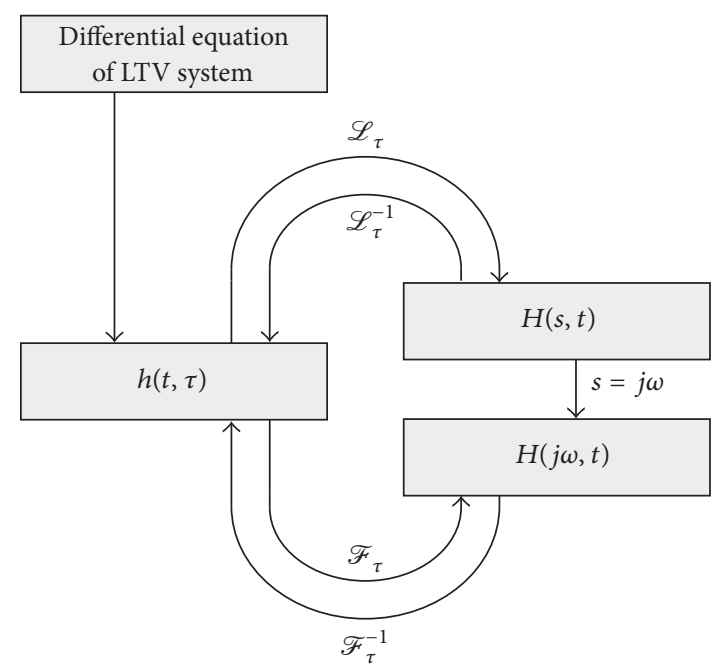

(b)

FIGURE 2: Relations between methods of description used for (a) LTI systems and (b) LTV systems.

equation with coefficients depending on time (4). The main problem of this description is that the solution to higherorder parametric equations usually, except for rare cases, does not exist.

The second method of LTV system expression in the time domain is a parametric convolution with an impulse response as a kernel [4]:

$$
y(t)=\int_{0}^{t} h(t, \tau) x(\tau) \mathrm{d} \tau
$$

where

$$
h(t, \tau) \text { is the system impulse response. }
$$

In contrast to classical stationary systems, the impulse response of an LTV system is a function of two variables: time and the moment of signal application to the input of a system.

The transfer function and the frequency response for LTV systems can be defined in various ways $[1,12,36-38]$. The most popular definitions have been given below:

$$
\begin{aligned}
H(s, t) & =\mathscr{L}_{\tau}\{h(t, \tau)\}, \\
H(j \omega, t) & =\mathscr{F}_{\tau}\{h(t, \tau)\}=\left.H(s, t)\right|_{s=j \omega},
\end{aligned}
$$

where

$\mathscr{L}_{\tau}, \mathscr{F}_{\tau}$, are the Laplace and Fourier transforms with respect to the variable $\tau$.

The functions $H(s, t)$ and $H(j \omega, t)$ are called transfer functions or frequency characteristics of LTV systems [1,37, 38]. They are very helpful representations used in the LTV system theory $[5,36,39]$.

The relations between various methods of LTI and LTV system description have been illustrated in Figure 2. The main difference consists in the lack of direct transition between differential equations and transfer functions for LTV systems. The impulse response function $h(t, \tau)$ of an LTV system can be obtained on the base of fundamental solutions of the differential equation describing this system in time domain. The determination of impulse response is essential and necessary to obtain the frequency characteristics of LTV filters. Further and detailed analysis has been limited to the first-order low-pass filters.

2.1. Model of the First-Order Low-Pass Filter. Analytical methods of solving higher-order differential equations with variable coefficients, with a few exceptions $[5,33]$, usually do not exist. The determination of the impulse response of LTV systems described by (4) is not generally possible.

Elementary first-order sections described in this work allow constructing more complex higher-order systems, which are stable, if the elementary sections are stable [40]. For the mentioned reasons, the consideration has been limited to the analysis of the LTV systems described by the first-order differential equation in the following form:

$$
y^{\prime}(t)+\omega(t) y(t)=c x(t),
$$

where

$x(t), y(t)$ are the input and output signals,

$\omega(t)$ is the parametric function,

$c$ is the constant gain coefficient, usually $c=\omega_{0}$, where $\omega_{0}$ denotes cutoff angular frequency of a stationary low-pass filter.

2.2. Parametric Function Variability. $\omega(t)$ is a parametric function and it can be interpreted as a cutoff angular frequency which depends on time. In the paper, the exponential and periodical waveform of the parameter has been assumed (Figure 3).

The mathematical description of the parametric function can be carried out in two ways. The first one consists in 


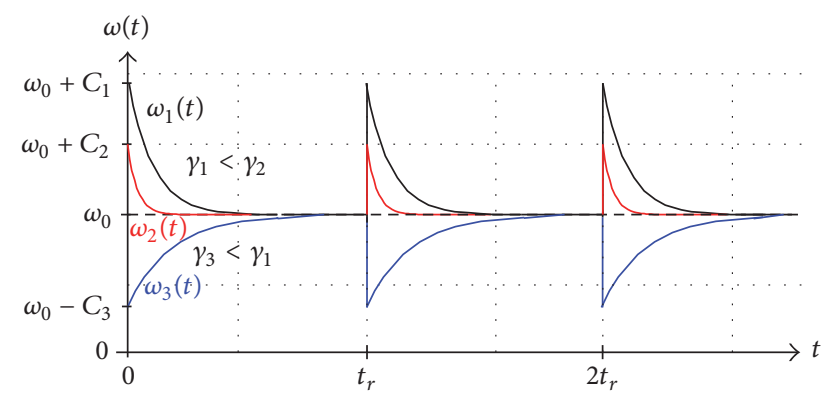

FIGURE 3: Examples of periodical exponential parametric function variability.

periodical repetition of the exponential function defined for a finite time interval which becomes the period. The second method uses the Fourier series.

Method 1. In the first period, parametric function is expressed by the equation:

$$
\begin{aligned}
& \omega_{1 P}(t)=\left(\omega_{0}+C e^{-\gamma t}\right) \cdot\left(\mathbf{1}(t)-\mathbf{1}\left(t-t_{r}\right)\right), \\
& \omega_{0}>0, C \in \mathrm{R}, \gamma \gg t_{r},
\end{aligned}
$$

where

$\omega_{0}$ is the cutoff angular frequency of a stationary prototype,

$C$ is the maximum or minimum value of $\omega(t)$,

$\gamma$ is the variability rate,

$t_{r}$ is the recurrence time,

$\mathbf{1}(t)$ is the unit step.

In the second step, the variation from the first time interval is generalized to the whole time axis:

$$
\begin{aligned}
\omega(t)= & \sum_{n=1}^{\infty} \omega_{1 P}\left(t-(n-1) t_{r}\right) \\
& \cdot\left(\mathbf{1}\left(t-(n-1) t_{r}\right)-\mathbf{1}\left(t-n t_{r}\right)\right) .
\end{aligned}
$$

In this way, we can obtain the expression describing the parametric function for $t \in \mathrm{R}^{+}$.

Method 2. The second way to express parametric functions uses the Fourier series. The considered function (Figure 3) fulfils the Dirichlet conditions; therefore it can be expressed as

$$
\begin{aligned}
& \omega(t) \\
& =\omega_{0}-\frac{C\left(e^{-\gamma t_{r}}-1\right)}{\gamma t_{r}} \\
& \quad+\sum_{h=1}^{\infty} \frac{2 C\left(1-e^{-\gamma t_{r}}\right)}{\sqrt{\left(\gamma t_{r}\right)^{2}+(2 h \pi)^{2}}} \cos \left(h \Omega t-\arctan \frac{2 h \pi}{\gamma t_{r}}\right),
\end{aligned}
$$

where

$$
\Omega=\frac{2 \pi}{t_{r}} .
$$

Both of the proposed methods can be used in further analysis. However, due to the Gibbs effect and difficult calculation using formula (10), the first method of description of parametric function (9) has been used in the next sections.

\section{Analysis of LTV System in Time Domain}

In the first stage of filter analysis, the solution $y_{1 P}(t)$ to differential equation (7) for the first period of parametric function was given. The general solution to (7) in a closed form is known and it is given by the formula [33]:

$$
\begin{aligned}
y_{1 P}(t) & =\omega_{0} y_{0}(t) e^{-\alpha(t)}+\omega_{0} \int_{0}^{t} e^{-\alpha(t)} e^{\alpha(\tau)} x(\tau) \mathrm{d} \tau, \\
y_{0} & =\left.y(t)\right|_{t=0}, \\
\alpha(t) & =\int_{0}^{t} \omega_{1 P}(t) \mathrm{d} t=\omega_{0} t+\frac{C}{\gamma}\left(1-e^{-\gamma t}\right) .
\end{aligned}
$$

For zero initial condition (13), (12) can be expressed in the form [41]:

$$
y_{1 P}(t)=\omega_{0} \int_{0}^{t} e^{-\omega_{0}(t-\tau)} e^{(C / \gamma)(\exp (-\gamma t)-\exp (-\gamma \tau))} x(\tau) \mathrm{d} \tau .
$$

From the comparison of (15) and the parametric convolution expressed by (5), it can be concluded that the impulse response of the considered system is given by

$$
h_{1 P}(t, \tau)=\omega_{0} e^{-\omega_{0}(t-\tau)} e^{(C / \gamma)(\exp (-\gamma t)-\exp (-\gamma \tau))} .
$$

Representing a part of statement (15) by its functional series, one gets

$$
e^{-(C / \gamma) \exp (-\gamma \tau)} \cong \sum_{k=0}^{n}(-1)^{k}\left(\frac{C}{\gamma}\right)^{k} \frac{e^{-k \gamma \tau}}{k !} .
$$

In the next step, using formulae (15) and (17), one can express the output signal in the first period of parametric function variability:

$$
\begin{aligned}
& y_{1 P}(t) \\
& =\omega_{0} \int_{0}^{t} e^{(C / \gamma) \exp (-\gamma t)} e^{-\omega_{0}(t-\tau)} \sum_{k=0}^{\mathrm{N}}(-1)^{k}\left(\frac{C}{\gamma}\right)^{k} \frac{e^{-\gamma k \tau}}{k !} \\
& \quad x(\tau) \mathrm{d} \tau .
\end{aligned}
$$

Then, one can generalize it for the remaining time intervals in such a form:

$$
\begin{aligned}
y(t)= & \sum_{n=1}^{\infty} y_{1 P}\left(t-(n-1) t_{r}\right) \\
& \cdot\left(\mathbf{1}\left(t-(n-1) t_{r}\right)-\mathbf{1}\left(t-n t_{r}\right)\right) .
\end{aligned}
$$




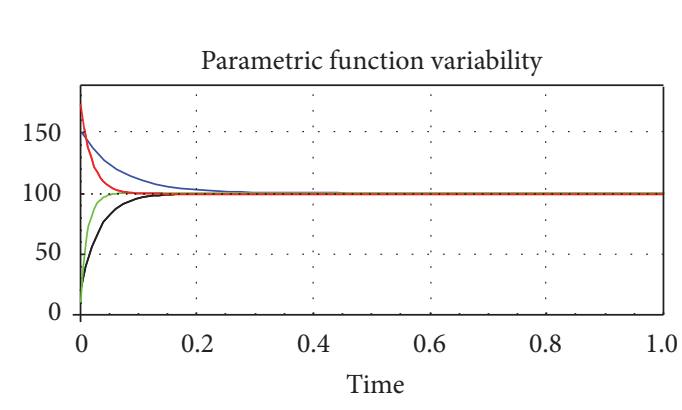

(a)

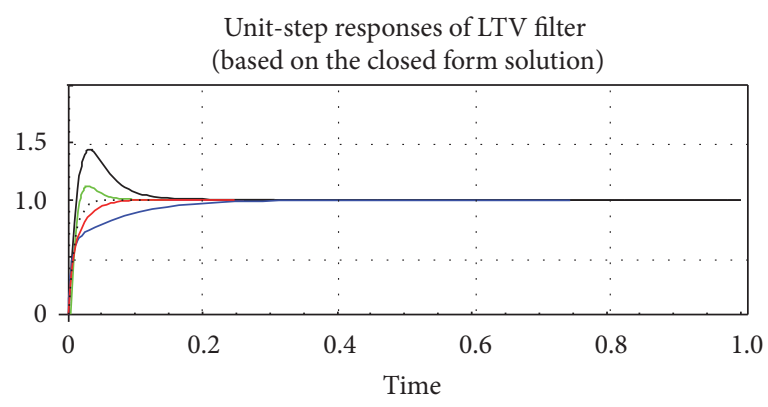

(b)

FIGURE 4: Calculation results: (a) waveforms of parametric function in the first period and (b) corresponding waveforms of output signal based on the closed form solution.

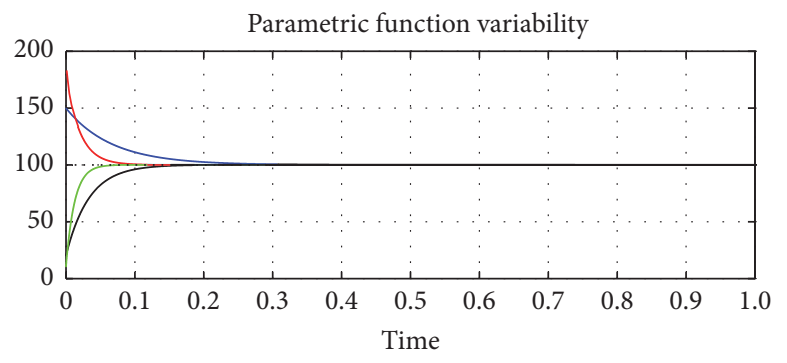

(a)

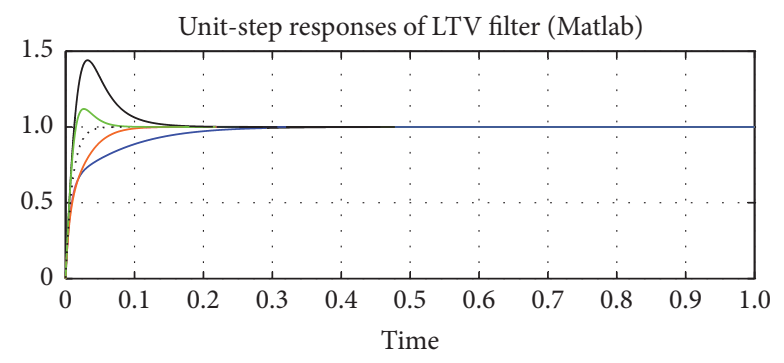

(b)

FIGURE 5: Matlab simulation results: (a) waveforms of parametric function in the first period and (b) corresponding waveforms of output signal.

In Figure 4, the parametric function variation (Figure 4(a)) and corresponding unit-step responses (Figure 4(b)) of a low-pass first-order LTV filter have been presented (the same colour has been used for the parametric function and the corresponding output signal).

Figures 4(a) and 4(b) have been drawn based on a mathematical analysis carried out in this work. In order to verify their correctness, the numerical solution to (7) in Matlab has also been arrived at. Results of Matlab simulation have been presented in Figure 5. It can be noticed that the output signal waveforms obtained by Matlab simulation are identical to those obtained by the mathematical analysis, which confirms the correctness of the considerations.

For an extensive class of input signals with limited energy or average power, the calculation of integral in (18) is possible and therefore the determination of the analytical solution to (7) in a closed form is possible.

\section{Example}

The first-order low-pass LTV filter presented in Figure 6 with a varying parameter $\omega(t)$ has been analyzed. The variability is expressed by (9). The waveforms of time-varying cutoff angular frequency $\omega(t)$ of the filter have been shown in Figure 7(a). A square signal with much lower frequency than the cutoff frequency of a stationary prototype of the considered LTV filter has been given on the input of the firstorder low-pass LTV filter:

$$
\omega_{\text {in }} \ll \omega_{0},
$$

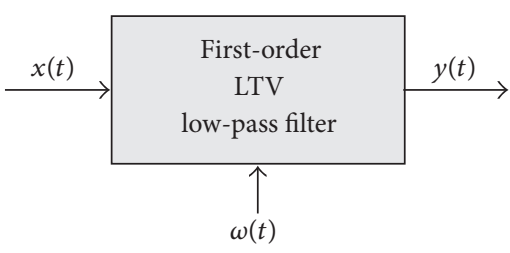

FIGURE 6: LTV filter model.

where

$\omega_{\text {in }}$ is the angular frequency of input signal, $\omega_{\text {in }}=$ $2 \pi f_{\text {in }}$.

On the base of the impulse response (16), it is possible to determine the filter response. The responses of the analyzed system for a few cutoff frequency variations (Figure 7(a)) have been presented in Figure 7(b). For comparison reasons, the response of a classical LTI filter (with a constant parameter $\omega_{0}$ ) to the square wave has also been included in Figure 7(b) (the dot line).

\section{Conclusions}

A closed form solution expressing the first-order LTV system impulse response for a class of periodic and exponential parametric functions has been presented in the paper. The first-order systems can be treated as elementary blocks which allow to construct more complex LTV systems. Moreover, 


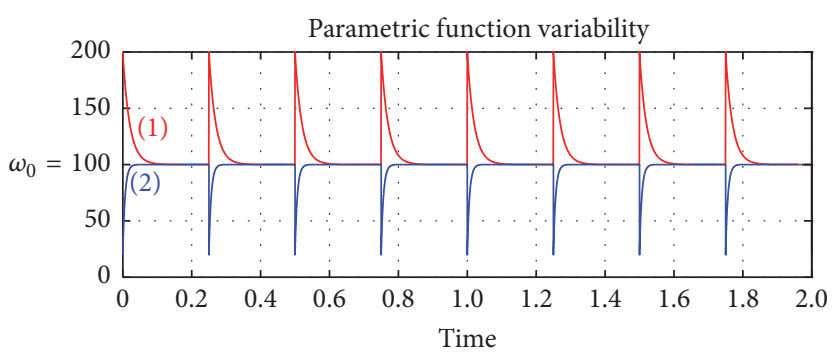

(a)

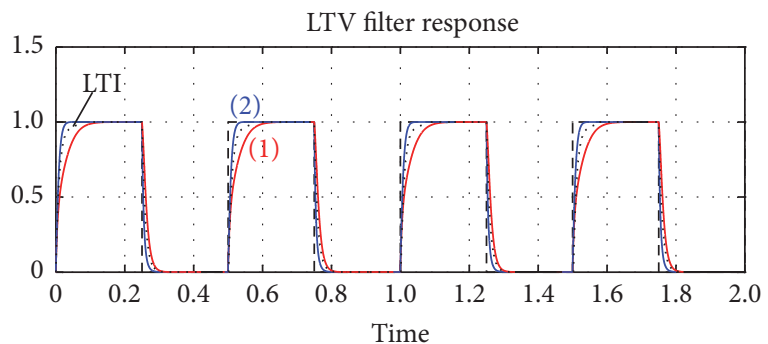

(b)

FIGURE 7: Result of LTV filter analysis: (a) waveforms of parametric function and (b) corresponding responses of the LTV system and the response of the LTI system.

only on the basis of the impulse response it is possible to determine the remaining time-frequency characteristics of the time-varying system under consideration. The obtained results showed that changes in the character of the parametric function variation lead to the modification of the properties of the system. It allows finding optimal structures and determining values of parameters for required filter properties (e.g., dynamic characteristics and noise reduction).

Time-varying filter parameters allow us to design more flexible systems for which the properties can be designed more freely. Changes in the waveform of the parameter can be used to shape the system response. For example, according to the variability of the parametric function, the cutoff frequency may be varied in time. In every period, when the parametric function achieves its steady value, the LTV system becomes an equivalent to a classical first-order low-pass filter with a constant value of the cutoff angular frequency.

\section{Conflicts of Interest}

The authors declare that they have no conflicts of interest.

\section{References}

[1] S. Erfani and N. Bayan, "On linear time-varying system characterizations," in Proceedings of the 2009 IEEE International Conference on Electro/Information Technology, EIT 2009, pp. 207-210, Windsor, ON, Canada, June 2009.

[2] M. Koksal and M. E. Koksal, "Commutativity of linear timevarying differential systems with nonzero initial conditions: a review and some new extensions," Mathematical Problems in Engineering, Article ID 678575, 25 pages, 2011.

[3] H. D’Angelo, Linear Time-Varying Systems. Analysis and Synthesis, Allyn and Bacon, Inc., Boston, Mass, USA, 1970.

[4] L. A. Zadeh, “Time-Varying Networks, I," Proceedings of the IRE, vol. 49, no. 10, pp. 1488-1503, 1961.

[5] J. A. Richards, Analysis of Periodically Time-Varying Systems, Springer-Verlag Berlin Heidelberg, New York, NY, USA, 1983.

[6] J.-P. Sferruzza, F. Chavrier, A. Birer, and D. Cathignol, "Numerical simulation of the electro-acoustical response of a transducer excited by a time-varying electrical circuit," IEEE Transactions on Ultrasonics, Ferroelectrics, and Frequency Control, vol. 49, no. 2, pp. 177-183, 2002.
[7] K. Schnell and A. Lacroix, "Model-based analysis of speech and audio signals for real-time processing based on time-varying lattice filters," in Proceedings of the 2009 IEEE International Conference on Acoustics, Speech, and Signal Processing, ICASSP 2009, pp. 3973-3976, Taipei, Taiwan, April 2009.

[8] H. Zhang, G. Bi, L. Zhao, S. G. Razul, and C.-M. S. See, "Timevarying filtering and separation of nonstationary FM signals in strong noise environments," in Proceedings of the 2014 IEEE International Conference on Acoustics, Speech, and Signal Processing, ICASSP 2014, pp. 4171-4175, Florence, Italy, May 2014.

[9] A. S. Mehr and T. Chen, "Representations of linear periodically time-varying and multirate systems," IEEE Transactions on Signal Processing, vol. 50, no. 9, pp. 2221-2229, 2002.

[10] G. Wang, "Time-varying discrete-time signal expansions as time-varying filter banks," IET Signal Processing, vol. 3, no. 5, pp. 353-367, 2009.

[11] S. Fuchs, M. Seguin, and A. Weisser, "Digital parametric filters for studio mixing desk," in Proceedings of the IEEE International Conference on Acoustics, Speech, and Signal Processing (ICASSP '82), vol. 7, pp. 97-100, IEEE, Paris, France, 1982.

[12] T. Claasen and W. Mecklenbrauker, "On stationary linear timevarying systems," IEEE Transactions on Circuits and Systems, vol. 29, no. 3, pp. 169-184, 1982.

[13] D. J. Shpak, "The analytical design of biquadratic parametric filters," in Proceedings of the IEEE Pacific Rim Conference on Communications, Computers and Signal Processing, pp. 649652, IEEE, Victoria, Canada, 1991.

[14] R. Kaszynski and J. Piskorowski, "Bessel filters with varying parameters," in Proceedings of the IEEE Instrumentationand Measurement Technology, vol. 1, pp. 757-761, IEEE, Ottawa, Canada, 2005.

[15] M. Jaskula and R. Kaszynski, "Using the parametric timevarying analog filter to average-evoked potential signals," IEEE Transactions on Instrumentation and Measurement, vol. 53, no. 3, pp. 709-715, 2004.

[16] R. Kaszynski and J. Piskorowski, "Selected structures of filters with time-varying parameters," IEEE Transactions on Instrumentation and Measurement, vol. 56, no. 6, pp. 2338-2345, 2007.

[17] T. M. Adami, R. Sabala, and J. J. Zhu, "Time-varying notch filters for control of flexible structures and vehicles," in Proceedings of the 22nd Digital Avionics Systems Conference (DASC '03), pp. 7.C.2-7.1-6, IEEE, Indianapolis, Ind, USA, October 2003.

[18] D. Grabowski, M. Maciążek, M. Pasko, and A. Piwowar, "Timeinvariant and time-varying filters versus neural approach applied to DC component estimation in control algorithms of 
active power filters," Applied Mathematics and Computation, 2017.

[19] L. Frackowiak, M. Gwozdz, and R. Porada, "Parametric filters in compensation of influence of nonlinear receiver on power network," in Proceedings of the IEEE International Symposium on Industrial Electronics (INSIE '96), vol. 2, pp. 608-611, IEEE, Warsaw, Poland, 1996.

[20] A. van Staveren, T. H. A. J. Cordenier, F. C. M. Kuijstermans et al., "The linear time-varying approach applied to the design of a negative-feedback class-B output amplifier," in Proceedings of the IEEE International Symposium on Circuits and Systems (ISCAS '99), pp. II-204-207, IEEE, Orlando, Fla, USA, June 1999.

[21] B. Ou and D. Liu, "Chaotic attractor generation via a simple linear time-varying system," Discrete Dynamics in Nature and Society. An International Multidisciplinary Research and Review Journal, Article ID 840346, 8 pages, 2010.

[22] K. Kluszczynski and J. Domin, "Two module electromagnetic launcher with pneumatic assist modelling, computer simulations and laboratory investigations," COMPEL - The International Journal for Computation and Mathematics in Electrical and Electronic Engineering, vol. 34, no. 3, pp. 691-709, 2015.

[23] A. N. Cheeran and P. C. Pandey, "Optimizing the sweep cycle of time-varying comb filters for binaural dichotic presentation in sensorineural hearing impairment," in Proceedings of the 14th International Conference on Digital Signal Processing, DSP 2002, pp. 1145-1148, Santorini, Greece, July 2002.

[24] J. Walczak and A. Romanowska, "Analysis of second order LTV section with exponentially varying parameters," Przeglad Elektrotechniczny, vol. 2, pp. 106-109, 2007.

[25] F. L. Neerhoff and P. van der Kloet, "A complementary view on time-varying systems," in Proceedings of the 2001 IEEE International Symposium on Circuits and Systems (ISCAS '01), vol. 3, pp. 779-782, IEEE, Sydney, Australia, May 2001.

[26] P. van der Kloet and F. L. Neerhoff, "Modal factorization of timevarying models for nonlinear circuits by the Riccati transform," in Proceedings of the 2001 IEEE International Symposium on Circuits and Systems (ISCAS '01), vol. 3, pp. 553-556, IEEE, Sydney, Australia, 2001.

[27] J. J. Zhu, "A unified spectral theory for linear time-varying systems-progress and challenges," in Proceedings of the 34th IEEE Conference on Decision and Control, pp. 2540-2546, IEEE, New Orleans, La, USA, 1995.

[28] J. J. Zhu and M. C. Mickle, "Synthesis of time-varying bandwidth filters based on all-pole LTI prototypes," in Proceedings of the 1998 American Control Conference, ACC 1998, pp. 28892894, Philadelphia, Pa, USA, June 1998.

[29] I. W. Sandberg, "Realization of a Class of Periodically Variable Systems," IRE Transactions on Circuit Theory, vol. 9, no. 4, pp. 416-417, 1962.

[30] G. Tohumoğlu and M. Köksal, "Steady state analysis of periodically time-varying networks by new developments in the spectral domain," International Journal of Circuit Theory and Applications, vol. 24, no. 4, pp. 519-528, 1996.

[31] M.-Y. Wu, "On stability of linear time-varying systems," International Journal of Systems Science. Principles and Applications of Systems and Integration, vol. 15, no. 2, pp. 137-150, 1984.

[32] M. Y. Wu, "A Note on Stability of Linear Time-Varying Systems," IEEE Transactions on Automatic Control, vol. 19, no. 2, p. 162, 1974.
[33] A. D. Polyanin and V. F. Zaitsev, Handbook of Exact Solutions for Ordinary Differential Equations, Chapman and Hall/CRC, Boca Raton, Fla, USA, 2nd edition, 2003.

[34] W. K. Chen, The Circuits and Filters Handbook, IEEE Press, New York, NY, USA, 1995.

[35] N. Bayan and S. Erfani, "Frequency analysis of time-varying systems," in Proceedings of the IEEE International Conference on Electro/Information Technology, pp. 33-36, East Lansing, Mich, USA, 2006.

[36] L. A. Zadeh, "Frequency Analysis of Variable Networks," Proceedings of the IRE, vol. 38, no. 3, pp. 291-299, 1950.

[37] A. Gersho, "Characterization of Time-Varying Linear Systems," Proceedings of the IEEE, vol. 51, no. 1, p. 238, 1963.

[38] A. Piwowar, "Time-frequency responses of generalized first order parametric sections," Archives of Electrical Engineering, vol. 64, no. 3, pp. 371-377, 2015.

[39] L. A. Pipes, "A mathematical analysis of a series circuit containing periodically varying resistance," IRE Transactions on Circuit Theory, vol. 2, no. 1, pp. 67-72, 1955.

[40] J. J. DaCunha, "Stability for time varying linear dynamic systems on time scales," Journal of Computational and Applied Mathematics, vol. 176, no. 2, pp. 381-410, 2005.

[41] A. Piwowar and J. Walczak, "Impulse responses of generalized first order LTV sections," Lecture Notes in Electrical Engineering, vol. 324, pp. 73-79, 2015. 


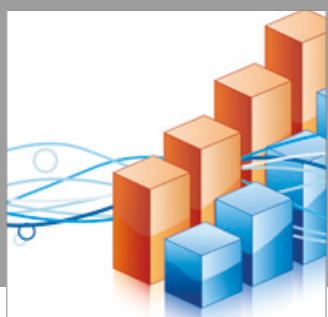

Advances in

Operations Research

vatersals

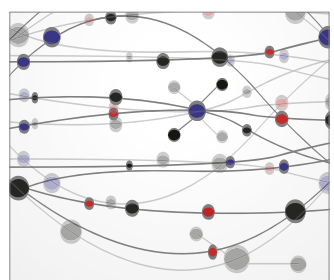

\section{The Scientific} World Journal
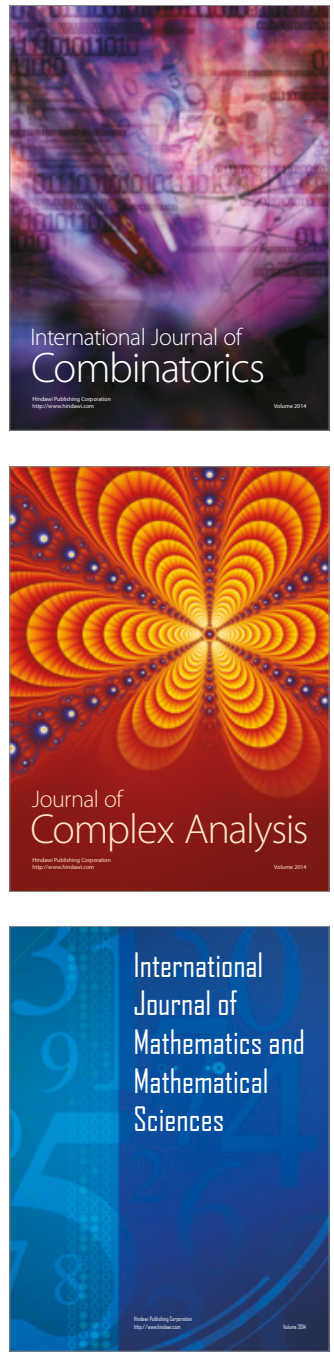
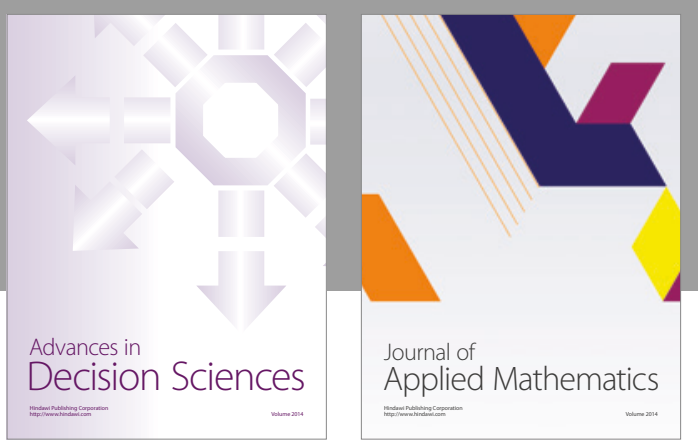

Algebra

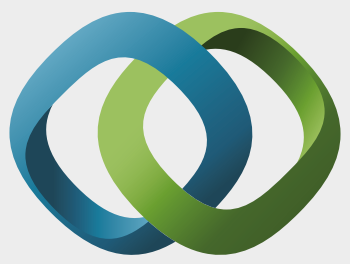

\section{Hindawi}

Submit your manuscripts at

https://www.hindawi.com
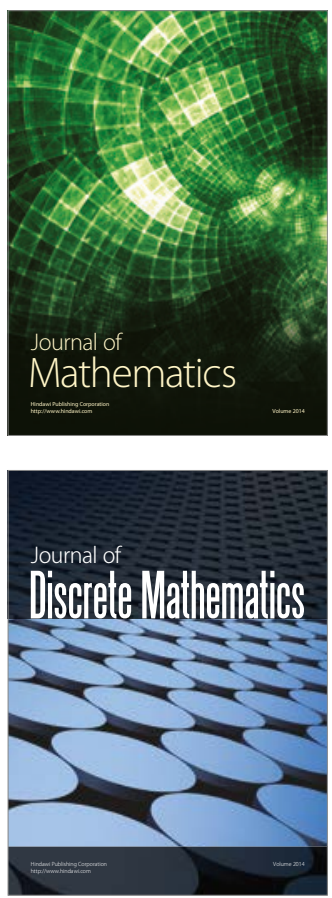

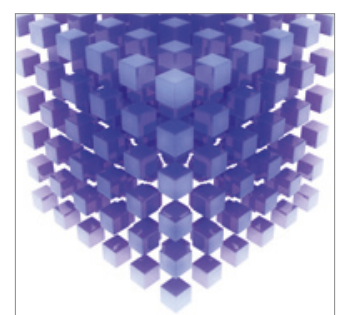

Mathematical Problems in Engineering
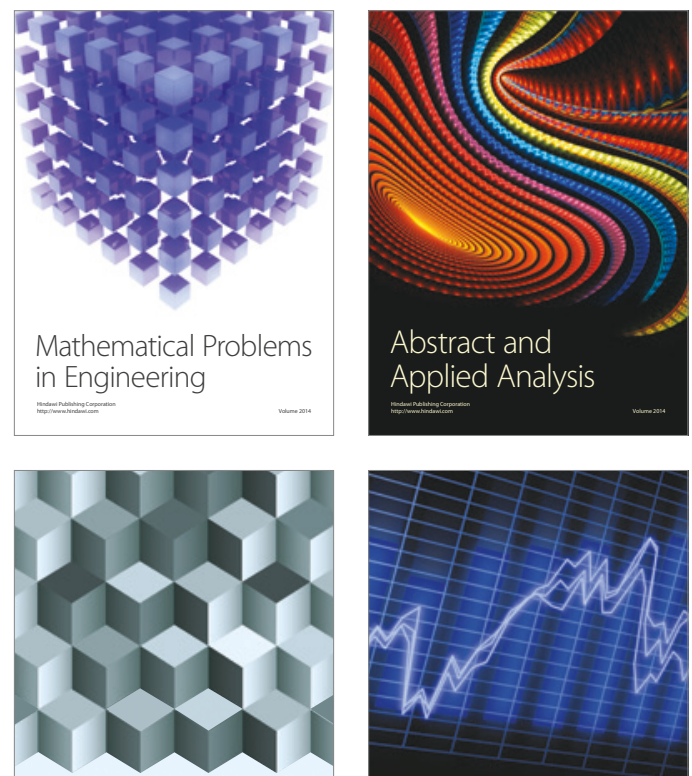

Journal of

Function Spaces

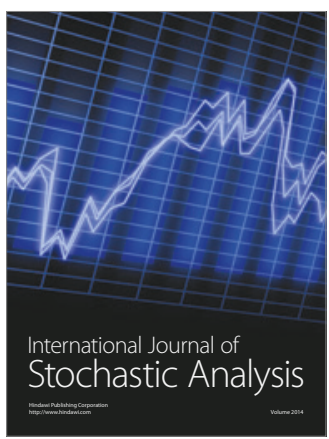

Probability and Statistics
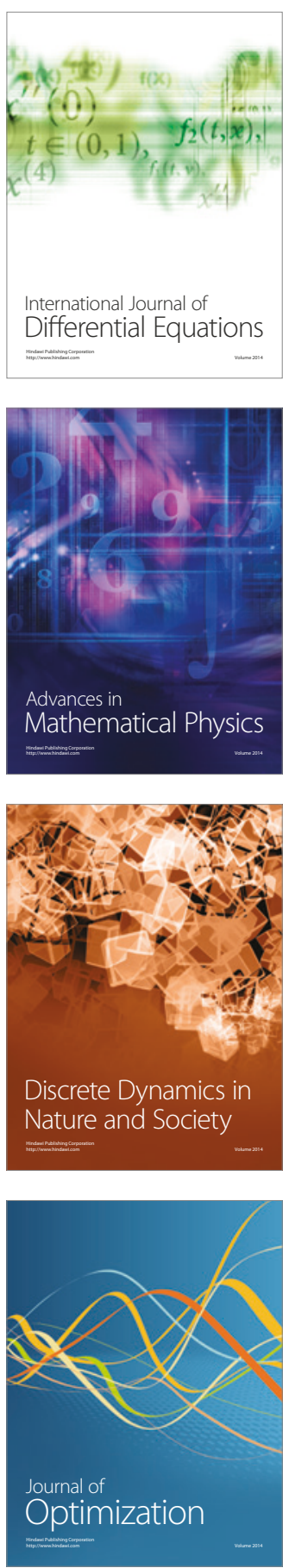